\title{
The Effect of Yoga on Women's Premenstrual Syndrome: A Randomized Controlled Clinical Trial
}

\author{
Mahin Kamalifard ${ }^{1}$, Abbas Yavari ${ }^{2}$, Mohammad Asghari-Jafarabadi ${ }^{3}$, Ghafoureh Ghaffarilaleh ${ }^{*}$, Ahmad \\ Kasb-Khah ${ }^{4}$
}

\begin{abstract}
Objectives: Women with premenstrual syndrome (PMS) show emotional, physical and behavioral symptoms regularly every month. Mild physiological symptoms of PMS appear in $95 \%$ of women at their reproductive age. We hypothesized to evaluate the effect of yoga exercise on PMS.

Materials and Methods: A randomized controlled clinical trial was conducted from April to October of 2015. Eligible women for inclusion were selected conveniently from women that had randomly referred to selected private obstetrics and gynecology clinics in Tabriz, Iran. Thereafter, 62 subjects were selected for the study. Subjects were randomly divided into 2 groups: 31 subjects for yoga and 31 subjects for control groups with blocks 4 and 6. The yoga group performed yoga for 10 weeks in 3 sessions with each session of 60 minutes duration. Subjects of control group did not do any yoga. Subjects completed the Premenstrual Symptoms Screening Tool (PSST) questionnaire for evaluation of the effect of yoga exercise on emotional, behavioral, physical symptoms and quality of life of subjects with PMS before and after the intervention. Data were analyzed by covariance.

Results: The mean \pm standard deviation (SD) of emotional, physical, behavioral symptoms and impact of yoga exercise on quality of life in yoga were $26.28 \pm 16.54,32.69 \pm 20.81,10.90 \pm 14.10,22.8 \pm 14.56$, and in the control group were $54.91 \pm 21.31,72.01 \pm 22.24$, $44.05 \pm 22.32,54.00 \pm 20.33$, respectively $(P<0.05)$.

Conclusion: Results highlight that yoga significantly relieves the PMS symptoms and can be prescribed for treatment of PMS.

Keywords: Premenstrual syndrome, Quality of life, Yoga
\end{abstract}

\section{Introduction}

Women with premenstrual syndrome (PMS) show majority of symptoms, particularly during luteal phase, regularly every month. These symptoms are classified into 3 emotional (EM), physical (PHY) and behavioral (BE) categories (1). The common symptoms of PMS are: bloating, breast tenderness, headache, frequent urination, loss of appetite, insomnia, irritability, depression, lack of energy and increased or decreased of libido $(1,2)$. Andrew et al (3) have shown that patients with PMS have lower work performance (27.5\%), impaired working relationships $(23.1 \%)$, disputation with their husbands $(8.82 \%)$, relationship problems with their children (6\%) and social relationship problems (41\%). PMS interferes with the life of patients directly by treatment expenditures and indirectly by inefficient working hours, absenteeism and productivity (4) and requires cure and management (5). Routinely, women with PMS use pharmacological (1) and non-pharmacological approaches (6) to decrease the severity of PMS symptoms. Whereas, prescribed medications (alprazolam, progesterone, fluoxetine and gonadotropin releasing hormone) have shown side effects such as exhaustion, insomnia, headaches, menstrual dysfunction, decreased sexual ability, nausea and vomiting
(1,7-9). Recently, yoga as a safe treatment has also been introduced (10).

Yoga is a Sanskrit word, meaning connection of the mind, body, emotions, logic and attention to the action. Yoga exercise is composed of physical (Asana), breathing (Pranayama) and mental (Pratt Hara) practices, resulting in health, relaxation and positive awareness. Yoga stretching practices with deep breathing and meditation strengthens muscles and protects the spine and joints of body (11). Evidences indicated that physical and mental health benefits of yoga occur through negative moderation of hypothalamic-pituitary-adrenal axis in association with sympathetic nervous system (SNS). Yoga exercise, negatively, via vagal nerves, regulates secretion of hormones (cortisol, glucose, plasma's renin, epinephrine and norepinephrine) into the bloodstream which are responsible for body functions (10). Yoga reduces the negative effects of induced stress to immune system by regulating positively the adjustment of immunoglobulin A (12). Additionally, yoga exercise reduces harmful inflammatory secretions, which make women with PMS, comfortable. Studies in India have shown positive impacts of yoga exercise on women with PMS on decreasing heart beats, blood pressure and anxiety $(6,13)$. 
With considering: $(a)$ the positive effects of physical exercises on global women's health (14-16), (b) the negative attitudes of the Iranian society towards women's sports (17), (c) the low percentage $(0.3 \%)$ of participation of women in sports in East Azerbaijan, Iran $(18,19),(d)$ lack of any kind of study from Iran on PMS and yoga, and (e) the effects of culture on PMS (20), the current investigation was designed to study the effects of yoga on women with PMS in reproductive age, in Tabriz society.

\section{Materials and Methods}

A controlled randomized clinical trial was conducted on women with PMS that had referred to private obstetrics and gynecology clinics in Tabriz, Iran from April to October of 2015. Randomly, 20 private obstetrics and gynecology clinics were selected using random numbers table. Researcher selected 150 subjects from referred patients to the private clinics complaining of PMS. Researcher, selected eligible subjects for the study based on objective criteria (convenience).

The names, phone numbers and addresses of subjects were recorded. To ensure PMS, Premenstrual Symptoms Screening Tool (PSST) questionnaire was provided to the subjects to complete them in two consecutive months. Subjects were requested to complete the demographic questionnaire as well. Two months after monitoring the patients using PSST questionnaire, 62 subjects with PMS entered the study. Eligible criteria included age between 20-45 years old, having PMS according to the PSST questionnaire, having regular menstrual periods, being non-athletic for duration of 3 months, not being under any treatment with chemical/herbal medications or oral contraceptives, having no depression and genital tract diseases, not drinking alcohol or smoking cigarettes, not to be a tobacco or illicit drug user, not having any joint diseases, rheumatoid arthritis or surgeries that could affect yoga exercise, not to be a caffeine user (expressed by subjects) and have a willingness to participate in the study. The subjects were excluded if they had no willingness to continue the investigation and/or have had experienced yoga exercise before.

Based on the research by Kanojia et al (21), the number of the subjects to be involved in the study was calculated by considering $95 \%$ interval confidence and $80 \%$ of power for 26 cases for yoga group $(-17.50 \pm 12.43)$ and 26 cases for control group $(-0.65 \pm 27.54)$. A $20 \%$ dropout was considered in cases, so 31 subjects were calculated per group. The allocation was done randomly for the yoga and the control group in the form of 4 and 6 blocks. To make the allocation blindness, researchers used opaque envelopes containing the names of the groups which were labeled with numbers. The envelopes were prepared by non-involved researcher. The first envelope was given to the first subject. Before allocation, the researcher or subjects were not aware of the allocation results. The researcher and analyzer were unaware until the end of the study. Totally, 31 subjects were assigned to yoga and 31 subjects to control groups.
A coach with valid certification in yoga, worked with the yoga group over a period of 10 weeks in 3 sessions for $60 \mathrm{~min} / \mathrm{wk}$. The first 2 weeks of the yoga exercise were just for physiological adaptations, then subjects continued the yoga for 10 weeks (22-24).

Researchers were in contact with control group subjects by phone calls. The first phone call was made 30 days after admission to the study and the second phone call was done after finishing the intervention in the yoga group. The aim of the phone calls was following the requirements of the control group and reminding them to fill out the questionnaires, asking about any probable using or used medications and filling out the PSST questionnaire at the end ( Figure 1).

Experimental Design and Statistical Analyses

The data collection tools were both demographic and PSST questionnaires. The demographic questionnaire consisted of age, education level, marital status and menstrual cycle. The PSST questionnaire was composed of 19 questions including 4 criteria namely: not at all, mild, moderate and severe, scoring from zero to 3. PSST questionnaire consisted of 2 categories; the first category had 14 questions about EM, PHY and BE symptoms and the second category had 5 questions considering the effects of PMS on quality of life. The total score was computed based on summing over the items which ranged between 0 to 100 for each category. The average score was calculated in each case. The questionnaire of PSST is rated at the University of McMaster in Canada. In Iran Hariri et al (25) standardized the PSST questionnaire in Iranian population. Both the reliability and validity index for PSST questionnaire are reported 0.7 and 0.8 , respectively. The validity of content was assessed by 10 experts specialized in sport sciences, midwifery and gynecology. The internal consistency of reliability was assessed by Cronbach $\alpha$. The values obtained were: EM 0.73, PHY 0.74, BE and the effects of PMS on quality of life were 0.94 and 0.73 , respectively. The PSST questionnaire was completed 3 times ( 2 times before yoga exercise intervention and one time after yoga exercise intervention).

Data was analyzed by IBM SPSS version 20.0 (IBM Corp. Armonk, NY, USA). Data was analyzed for frequencies, percentages, and quantitative variables with mean \pm SD. Normality of the quantitative variables were assessed using Kolmogorov-Smirnov test. Intentionto-treat was applied (87\% of ITT: Yoga, $\mathrm{n}=26$; Control, $\mathrm{n}=28$; total 54 ). All of variables were normal. The basic and demographic variables were analyzed by chi-square and student $t$ test. Paired $t$ test was used for within-group comparisons. For analyzing the covariance, the effect of the yoga intervention was measured through comparison and adjustments on the baseline. Finally, results were considered with a $95 \%$ CI.

\section{Results}

Of the 150 women assessed for eligibility, finally 62 women were randomized in the yoga (31 subjects) and 


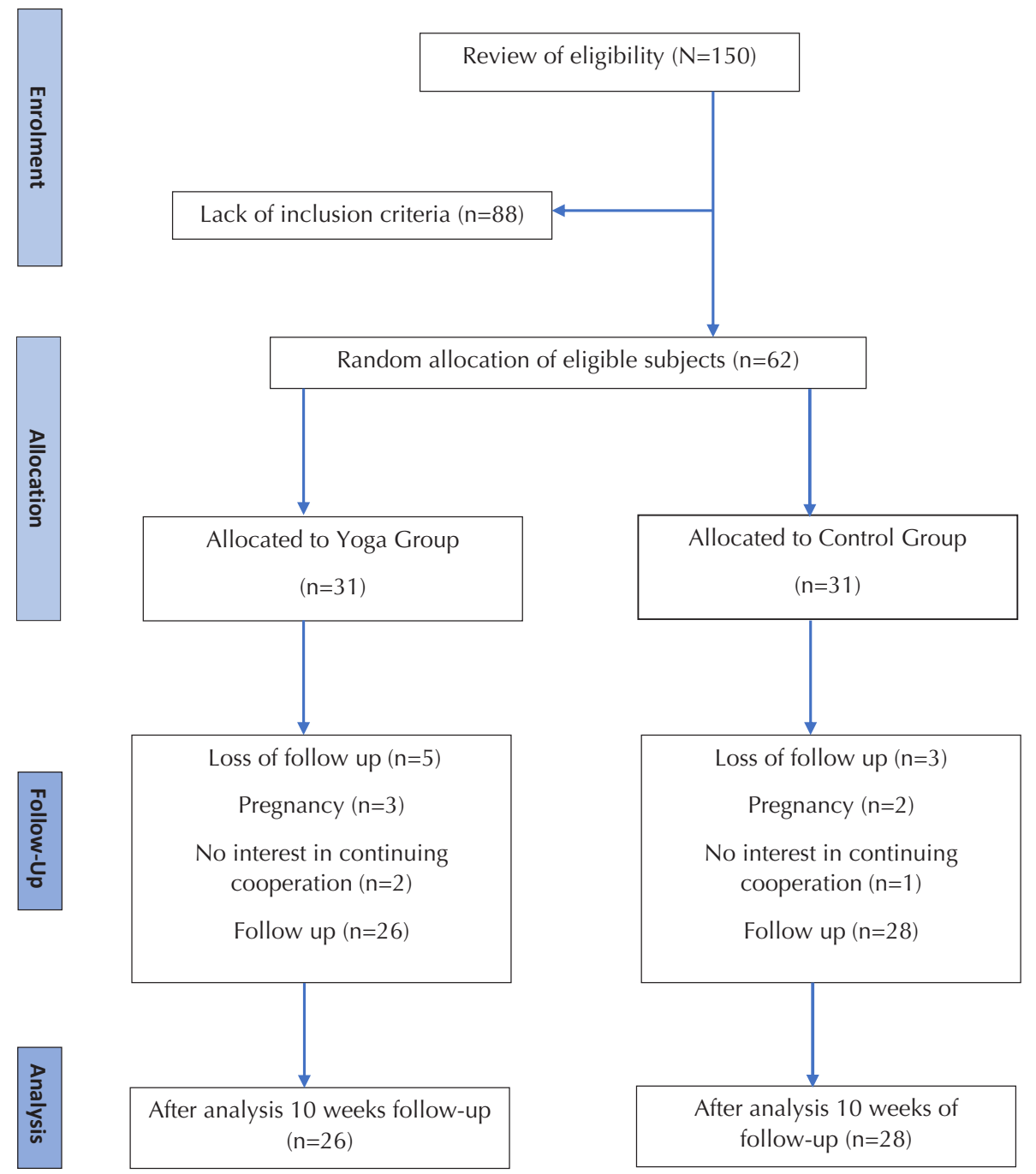

Figure 1. Flowchart of the Subjects Through Each Stage of the Trial.

control (31 subjects) groups. Some of the subjects left the study because of becoming pregnant ( 3 in the yoga and 2 in the control) or not being willing to continue further (2 in the yoga and 1 in the control) (Figure 1).

The mean \pm SD for age was $34.4 \pm 5.3$ and $30.1 \pm 6.2$ for yoga and control groups, respectively. Both yoga and control groups were similar in means in terms of the demographic characteristics, except employment status. Comparisons for the demographic characteristics of the subjects in each group are provided in Tables 1 and 2.

The variables were compared between yoga and control groups before and after intervention (Table 3).

Results showed no significant difference in both yoga and control groups before intervention for EM, PHY and $\mathrm{BE}$ variables. However, after intervention there was a significant difference in all EM, PHY and BE variables between yoga and control groups $(P<0.001)$.

There was no significant difference on the "impact of PMS on the life" before applying intervention in both yoga and control groups; however after yoga intervention there were significant differences $(P<0.001)$.
Furthermore, data analysis by paired $t$ test showed no significant difference before and after intervention in the control group.

\section{Discussion}

The current study highlighted that yoga decreases the symptoms of PMS in women. Results from this study confirm the previous outcomes of physical and aerobic exercises that showed a reduction in the symptoms of PMS (26-30).

Nowadays, western remedies beside the complementary and alternative medicines are not able to provide enough satisfactions for women with PMS $(9,31,32)$.

Studies have indicated that yoga decreases the different symptoms of PMS affecting positively the effects of yoga on relaxation and controlling of the nervous system. In a study, it has been shown that Hatha yoga increases relaxation of the mind and body, reduces tension of the muscles and stress, which subsequently improves sleeping with less anxiety. This study examined vital symptoms and showed Hatha yoga exercise reduces sympathetic activity, 
Table 1. Demographic Characteristics of the Subjects of Yoga and Control Groups

\begin{tabular}{|c|c|c|c|c|}
\hline Variables & & $\begin{array}{l}\text { Control } \\
(n=28)\end{array}$ & $\begin{array}{c}\text { Yoga } \\
(n=26)\end{array}$ & $P$ Value $^{\mathrm{a}}$ \\
\hline \multirow{2}{*}{$\begin{array}{l}\text { Job } \\
\text { statement }\end{array}$} & Housekeeper & $4(14.3)$ & $11(42.3)$ & \multirow{2}{*}{0.033} \\
\hline & Practitioner & $24(85.7)$ & $15(57.7)$ & \\
\hline \multirow{3}{*}{$\begin{array}{l}\text { Marital } \\
\text { status }\end{array}$} & Single & $11(39.3)$ & $12(46.2)$ & \multirow{3}{*}{0.574} \\
\hline & Married & $15(53.6)$ & $14(53.8)$ & \\
\hline & Divorced & $2(7.1)$ & $0(0)$ & \\
\hline \multirow{3}{*}{$\begin{array}{l}\text { Number of } \\
\text { children }\end{array}$} & 0 & $16(57.1)$ & $15(57.7)$ & \multirow{3}{*}{0.642} \\
\hline & 1 & $9(32.1)$ & $6(23.1)$ & \\
\hline & 2 & $3(10.7)$ & $5(19.2)$ & \\
\hline \multirow{4}{*}{$\begin{array}{l}\text { Education } \\
\text { statement }\end{array}$} & Diploma & 5 (17.9) & $3(11.5)$ & \multirow{4}{*}{0.473} \\
\hline & Associate degree & $3(10.7)$ & $1(3.8)$ & \\
\hline & Bachelor & $18(64.3)$ & $17(65.4)$ & \\
\hline & Master & $2(7.1)$ & $5(19.2)$ & \\
\hline \multirow{3}{*}{$\begin{array}{l}\text { Body mass } \\
\text { index }\end{array}$} & $\leq 25$ & $20(74.1)$ & $15(57.7)$ & \multirow{3}{*}{0.399} \\
\hline & $25.1-30$ & $5(18.5)$ & $9(34.6)$ & \\
\hline & $<30.1$ & $2(7.4)$ & $2(7.7)$ & \\
\hline
\end{tabular}

a $P$ value denotes chi-square tests.

Table 2. Comparison of the Mean of the Demographic Characteristics in the Yoga and Control Groups

\begin{tabular}{lccl}
\hline Time & $\begin{array}{c}\text { Control }(\mathbf{n}=\mathbf{2 8}) \\
\text { (Mean } \pm \text { SD) }\end{array}$ & $\begin{array}{c}\text { Yoga }(\mathbf{n}=\mathbf{2 6}) \\
\text { (Mean } \pm \text { SD) }\end{array}$ & P Value $^{\mathrm{a}}$ \\
\hline Age & $30.179 \pm 6.290$ & $34.462 \pm 5.368$ & 0.010 \\
Period duration & $6.214 \pm 1.371$ & $6.692 \pm 2.074$ & 0.319 \\
Period Time & $28.821 \pm 2.539$ & $29.923 \pm 2.799$ & 0.135 \\
Age of first period & $13.357 \pm 1.747$ & $13.115 \pm 1.306$ & 0.570 \\
\hline
\end{tabular}

a $P$ value denotes independent $t$ test. stress pressure and increases relaxation of women with PMS (30).

Rani et al (29) in a study on women with irregular menstruation, have shown that yoga Nidra significantly decreases any pain caused due to gastrointestinal and urogenital problems, or cardiovascular disorders. Furthermore, Rani et al (28) in a study on women with irregular menstruation, acute anxiety and severe depression, have shown that yoga exercise cannot significantly improve severe symptoms but rather mild symptoms. Dvivedi et al (30) have shown that sedation exercise can improve symptoms of PMS caused from stress. These researchers used electromyograms to measure muscle tension and stress that were stored in the form of muscle tension. Patients who performed yoga exercise experienced a reduction in muscle tension because of reduction in stress. Stress-related disorders such as increased activity of the sweat glands, are shown to decrease by yoga exercise. Furthermore, the effects of yoga exercise might be related to its functions that involves the person's mind and body and makes the person calm and relax.

In the current study, the symptoms of PMS decreased in the yoga group including anger or irritability, anxiety or stress, crying or rising sensitivity to negative issues, depressed mood or hopelessness, loss of interest to work activities, decreased interest to house activities, loss of interest to social activities, concentration problems or lack of focus on the lesson. Dvivedi et al (30) reported that $80 \%$ of subjects doing yoga exercise have shown improvement in the symptoms of irritability. In contrast with the current study, Safavi-Naeeni (26) reported that by doing both aerobic and physical exercises, symptoms of PMS showed less positive effects compared to the control group. These researchers recommended that a fitness exercise might reduce the symptoms of PMS.

Recent studies highlighted that a regular exercise habit

Table 3. Variables of EM, PHY, BE and the Effects of PMS on the Quality of Life Between Yoga and Control Groups

\begin{tabular}{|c|c|c|c|c|}
\hline \multirow{2}{*}{ Variable } & \multirow{2}{*}{ Time } & \multirow{2}{*}{$\begin{array}{l}\text { Yoga }(n=26) \\
\text { Mean } \pm \text { SD }\end{array}$} & \multirow{2}{*}{$\begin{array}{l}\text { Control }(n=28) \\
\text { Mean } \pm \text { SD }\end{array}$} & \multirow{2}{*}{$P$ Value $^{\mathrm{a}}$} \\
\hline & & & & \\
\hline \multirow{3}{*}{ Emotional (EM) } & Before intervention & $62.34 \pm 16.26$ & $54.32 \pm 19.16$ & 0.11 \\
\hline & After intervention & $26.28 \pm 16.54$ & $54.91 \pm 21.31$ & $<0.001$ \\
\hline & $P$ value $^{\mathrm{b}}$ & $<0.001$ & 0.856 & \\
\hline \multirow{3}{*}{ Physical (PHY) } & Before intervention & $71.15 \pm 22.39$ & $78.57 \pm 14.95$ & 0.155 \\
\hline & After intervention & $32.69 \pm 20.81$ & $72.01 \pm 22.24$ & $<0.001$ \\
\hline & $P$ value $^{\mathrm{b}}$ & $<0.001$ & 0.077 & \\
\hline \multirow{3}{*}{ Behavioral (BE) } & Before intervention & $45.51 \pm 19.89$ & $44.04 \pm 18.54$ & 0.078 \\
\hline & After intervention & $10.90 \pm 14.10$ & $44.05 \pm 22.32$ & $<0.001$ \\
\hline & $P$ value $^{\mathrm{b}}$ & $<0.001$ & 1 & \\
\hline \multirow{3}{*}{$\begin{array}{l}\text { Impact of PMS on the } \\
\text { quality of life }\end{array}$} & Before intervention & $58.79 \pm 14.61$ & $54.85 \pm 14.68$ & 0.327 \\
\hline & After intervention & $22.8 \pm 14.56$ & $54.00 \pm 20.33$ & $<0.001$ \\
\hline & $P$ value $^{\mathrm{b}}$ & $<0.001$ & 0.711 & \\
\hline
\end{tabular}

a $P$ value in ANCOVA test

${ }^{\mathrm{b}} P$ value in T-test Paired Samples

Note: Controlled potential confounders were: employment status, age and education. 
probably decreases some physical and psychological PMS symptoms. Pain is one of the common symptoms of PMS that affects mood and behaviour of the women with PMS by promoting different brain regions during cognitive tasks (33). It has been described that a short-term yoga in women with PMS improved attention and women felt better (34). Furthermore, yoga exercise by inducing an enhancement in alpha-brain-wave production causes slower abdominal breathing (33). Yoga affects positively the activity of brain wave where alpha-brain waves are associated with peace, relaxation, mood, and secretion of serotonin helping patients to feel relaxed after yoga intervention. Moreover, a reduction in the symptoms of PMS, increases with exercise of yoga as well (27).

Additionally, a study in India using randomized controlled trial emphasized that yoga Nidra improved hormone disorders in dysmenorrhea, oligomenorrhea, menorrhagia, metrorrhagia, and hypomenorrhea (27). Yoga poses such as cobra, cat, and fish reduce the severity and duration of primary dysmenorrhea (35). The severity of dysmenorrhea disorder and serum homocysteine concentrations decrease 8 weeks after yoga exercise (36).

Dvivedi et al (30) concluded that during premenstrual cycle the weight of breasts increased by $83 \%$, abdominal pain by $76 \%$, back pain and vaginal discharge by 67 and $53 \%$, respectively. These authors, using relaxation therapy, showed higher relaxation and lower sympathetic activity. During stress, heartbeats, systolic and diastolic blood pressure, muscle tension, skin contractions and respiration were increased, whereas parasympathetic activity was decreased. They indicated that negative effects of PMS using yoga exercise reduced with relaxation poses. Similarly, the current study showed a significant decrease in physical symptoms of PMS $(P<0.001$; Table 3). Furthermore, it be noted that slowly stretched muscles decrease muscle pain and body pose affected by PMS. Indeed, yoga exercise by strengthening muscles, increasing flexibility of the muscles, and facilitating the diaphragmatic breathing, regulates the functions of the muscles in viscera, glands, blood vessels, nerves and consequently lymphatic circulation.

On the other hand, the current study showed that yoga exercise significantly improved the behavioral symptoms of PMS such as overeating or food cravings, insomnia, oversleeping or desire to sleep, feeling of confusion or uncontrollable behaviors $(P<0.05)$. Newham et al (37) described that yoga exercise reduced anxiety and feeling of confusion in pregnant women. However, yoga exercise affects the appetite and insomnia of patients with breast cancer $(38,39)$. On the other hand, Vadiraja et al found a positive correlation between physical and mental symptoms such as exhaustion, fatigue, nausea and vomiting, pain, shortness of breath, insomnia, loss of appetite and constipation in the patients with breast cancer. They explained yoga exercise significantly decreases the negative effects of cancer (38).

It has been shown that PMS affects the quality of life. Women with PMS may see negative effects of PMS at their job or school performance, relationship with colleagues and friends, relationship with husband and children, social activities and family responsibilities. In the current study, subjects expressed that the quality of their life improved by doing yoga exercise $(P<0.05)$. Accordingly, Woodyard et al (40) stressed that continued yoga exercise improved the quality of life, self-awareness and having positive energy to stay stable and happy. Moreover, yoga exercise can cause a physiological statement and provide a feeling of balance and unity between mind and body (41).

Since PMS without treatment can cause problems and affect the quality of life of women, it is essential to look for tools and treatments that can relieve the symptoms of PMS that are affordable in individual. The results showed that yoga is a peaceful and effective approach for decreasing PMS symptoms. Therefore, yoga exercise is recommended for women with PMS as it does not have any side effects and is safe. Women can do yoga exercise by themselves at any time and any place (42-44).

\section{Limitations}

All the subject's responses in the current study were considered to be honest and right. Detection of the accuracy of the responses collected from subjects were beyond the researcher's capacity.

\section{Strengths}

The researcher and analyzer were unaware until the end of the study. Additionally, as obstetrics and gynecology private clinics were randomly selected, the result can be generalized to Iranian women society.

\section{Suggestions for Future Studies}

The effect of yoga exercise on hormones and blood metabolites concentrations are not studied enough and concentrations should be investigated before and after yoga intervention.

\section{Conclusion}

In conclusion, yoga exercise reduced the symptoms of PMS in women with PMS during regular reproductive cycle. Consequently, quality of life of women with PMS considerably increased as the symptoms of PMS decreased and women felt calmer with less pain. Therefore, medical therapy might be just needed in severe situation.

\section{Ethical Issues}

The required approvals were documented from the ethical committees of Tabriz University of Medical Science (No. 93184). Then documents were submitted to Iranian Registry of Clinical Trials (http://irct.ir/, identifier: IRCT201501216582N9). Participation of women with PMS in the present study was voluntary, where they were selected randomly. The aim was explained to the researcher and the objectives and methods of the study to all subjects. Thereafter, written informed consent and oral assent was obtained from all subjects who agreed to participate. All procedures of gathering and managing of 
data were done carefully and anonymously, considering the security of the data.

\section{Conflict of Interests}

The authors have no conflict of interest to disclose.

\section{Financial Support}

This study has been supported by a grant from Tabriz University of Medical Science.

\section{Acknowledgments}

We would like to thank the personnel of the Tabriz University of Medical Science in the current study. We also would appreciate the honest efforts of Mrs. Leila Alibaf for supervising the yoga group.

\section{References}

1. Burkman RT. Berek \& Novak's Gynecology. JAMA 2012;308(5):516-7.

2. Zaafrane F, Faleh R, Melki W, Sakouhi M, Gaha L. [An overview of premenstrual syndrome]. J Gynecol Obstet Biol Reprod (Paris) 2007;36(7):642-52. French.

3. Andrews G. Women's Sexual Health. UK: Elsevier Health Sciences; 2005.

4. Ledger WL. The Menstrual Cycle. Dewhurst's Textbook of Obstetrics \& Gynaecology. Wiley-Blackwell; 2012:485-494.

5. Kelderhouse K, Taylor JS. A review of treatment and management modalities for premenstrual dysphoric disorder. Nurs Womens Health. 2013;17(4):294-305.

6. Chen KM, Tseng WS, Ting LF, Huang GF. Development and evaluation of a yoga exercise programme for older adults. J Adv Nurs. 2007;57(4):432-441.

7. Newcombe S. Stretching for health and well-being: Yoga and women in Britain, 1960-1980. Asian Med. 2007;3:3763.

8. Saper RB, Eisenberg DM, Davis RB, Culpepper L, Phillips RS. Prevalence and patterns of adult yoga use in the United States: results of a national survey. Altern Ther Health Med. 2003;10(2):44-49.

9. Dimmock PW, Wyatt KM, Jones PW, O'Brien PM. Efficacy of selective serotonin-reuptake inhibitors in premenstrual syndrome: a systematic review. Lancet. 2000;356(9236):1131-1136.

10. Sterling P. Principles of Allostasis: Optimal Design, Predictive Regulation, Pathophysiology, and Rational therapeutics. In: Schulkin J, ed. Allostasis, Homeostasis, and the Costs of Physiological Adaptation. Cambridge: Cambridge University Press; 2004.

11. McEwen BS, Karatsoreos IN. Sleep deprivation and circadian disruption: stress, allostasis, and allostatic load. Sleep Med Clin. 2015;10(1):1-10. doi:10.1016/j.jsmc.2014.11.007.

12. Brotto LA, Krychman M, Jacobson P. Eastern approaches for enhancing women's sexuality: mindfulness, acupuncture, and yoga (CME). J Sex Med. 2008;5(12):2741-2748. doi: 10.1111/j.1743-6109.2008.01071.x.

13. Michalsen A, Grossman P, Acil A, et al. Rapid stress reduction and anxiolysis among distressed women as a consequenceof a three-month intensive yoga program. Med Sci Monit. 2005;11(12):CR555-561.

14. Júnior A, de Sousa MdSC, Miguel H. The psychological health of women after 16 weeks of practicing different exercise programs. JEP Online. 2015;18(2):32-44.

15. Karinkanta S, Nupponen R, Heinonen A, et al. Effects of exercise on health-related quality of life and fear of falling in home-dwelling older women. J Aging Phys Act. 2012;20(2):198-214.

16. Aparicio-Ting FE, Farris M, Courneya KS, Schiller A, Friedenreich CM. Predictors of physical activity at 12 month follow-up after a supervised exercise intervention in postmenopausal women. Int J Behav Nutr Phys Act. 2015;12:55. doi: 10.1186/s12966-015-0219-z.

17. Mirsafian H, Dóczi T, Mohamadinejad A. Attitude of Iranian female university students to sport and exercise. Iranian Studies. 2014;47(6):951-966.

18. Mozafari S, Gharah M. Sport for all in iran and comparing with some selected countries. Journal Of Movement Science \& Sports. 2006;3(6):151-171.

19. Amraee H, Safania AM, Farzan F. Study of barriers and challenges for recreational and sport activities of students Mazandaran universities from faculty members of physical education and exercise science of Mazandaran province viewpoint (with strategic approach). Int J Sport Stud. 2013;3(11):1164-1171.

20. Johnson TM. Premenstrual syndrome as a western culturespecific disorder. Cult Med Psychiatry. 1987;11(3):337-356.

21. Kanojia S, Sharma VK, Gandhi A, Kapoor R, Kukreja A, Subramanian SK. Effect of yoga on autonomic functions and psychological status during both phases of menstrual cycle in young healthy females. J Clin Diagn Res. 2013;7(10):2133-2139. doi: 10.7860/JCDR/2013/6912.3451.

22. Long R, Macivor C. The Key Muscles of Yoga. India: Bandha Yoga Publications LLC ; 2009.

23. Sulochana DT. Understanding Yoga Through Body Knowledge. India: Padmagandha; 2003.

24. Long R. The Key Poses of Yoga. India: Bandha Yoga; 2009.

25. Hariri FZ, Moghaddam-Banaem L, Bazi SS, Malehi AS, Montazeri A. The Iranian version of the Premenstrual Symptoms Screening Tool (PSST): a validation study. Arch Womens Ment Health. 2013;16(6):531-537. doi: 10.1007/ s00737-013-0375-6.

26. Safavi-Naeeni K. Comparison of the effects of exercise on aerobic and physical symptoms of premenstrual syndrome in women, city of Shiraz (Persian). Medical Science Journal of Islamic Azad University. 2008;18:177-80.

27. Rani M, Singh U, Agrawal GG, et al. Impact of Yoga Nidra on menstrual abnormalities in females of reproductive age. J Altern Complement Med. 2013;19(12):925-929. doi: 10.1089/acm.2010.0676.

28. Rani K, Tiwari S, Singh U, Singh I, Srivastava N. Yoga Nidra as a complementary treatment of anxiety and depressive symptoms in patients with menstrual disorder. Int J Yoga. 2012;5(1):52-56. doi: 10.4103/0973-6131.91715.

29. Rani K, Tiwari S, Singh U, Agrawal G, Srivastava N. Sixmonth trial of yoga nidra in menstrual disorder patients: effects on somatoform symptoms. Ind Psychiatry J. 2011;20(2):97-102. doi: 10.4103/0972-6748.102489.

30. Dvivedi J, Dvivedi S, Mahajan K, Mittal S, Singhal A. Effect of '61-points relaxation technique' on stress parameters in premenstrual syndrome. Indian J Physiol Pharmacol. 2008;52(1):69-76.

31. Beiranvand SP, Beiranvand NS, Moghadam ZB, et al. The effect of Crocus sativus (saffron) on the severity of premenstrual syndrome. Eur J Integr Med. 2016;8(1):55-61. 
32. Abdollahifard $\mathrm{S}$. The effects of vitamin $\mathrm{B} 1$ on the mental and behavioral symptoms related to premenstrual syndrome. J Psychosomatic Res. 2015;78(6):588.

33. Wu WL, Lin TY, Chu IH, Liang JM.The acute effects of yoga on cognitive measures for women with premenstrual syndrome. J Altern Complement Med. 2015;21(6):364-369. doi: 10.1089/acm.2015.0070.

34. Desai R, Tailor A, Bhatt T. Effects of yoga on brain waves and structural activation: a review. Complement Ther Clin Pract. 2015;21(2):112-128.

35. Rakhshaee Z. Effect of three yoga poses (cobra, cat and fish poses) in women with primary dysmenorrhea: a randomized clinical trial. J Pediatr Adolesc Gynecol. 2011;24(4):192-6. doi:10.1016/j.jpag.2011.01.059.

36. Chien LW, Chang HC, Liu CF. Effect of yoga on serum homocysteine and nitric oxide levels in adolescent women with and without dysmenorrhea. J Altern Complement Med. 2013;19(1):20-23. doi:10.1089/acm.2011.0113.

37. Newham JJ, Wittkowski A, Hurley J, Aplin JD, Westwood M. Effects of antenatal yoga on maternal anxiety and depression: A randomized controlled trial. Depress Anxiety. 2014;31(8):631-640. doi:10.1002/da.22268.

38. Vadiraja SH, Rao MR, Nagendra RH, et al. Effects of yoga on symptom management in breast cancer patients: A randomized controlled trial. Int J Yoga. 2009;2(2):73-79. doi:10.4103/0973-6131.60048.

39. Mustian KM. Yoga as treatment for insomnia among cancer patients and survivors: a systematic review. Eur Med J Oncol. 2013;1:106-115.

40. Woodyard C. Exploring the therapeutic effects of yoga and its ability to increase quality of life. Int J Yoga. 2011;4(2):4954. doi:10.4103/0973-6131.85485.

41. Arora S, Bhattacharjee J. Modulation of immune responses in stress by Yoga. Int J Yoga. 2008;1(2):45-55. doi: 10.4103/0973-6131.43541.

42. Büssing A, Michalsen A, Khalsa SBS, Telles S, Sherman KJ. Effects of yoga on mental and physical health: a short summary of reviews. Evid Based Complement Alternat Med. 2012;2012:165410.

43. Patel NK, Newstead AH, Ferrer RL. The effects of yoga on physical functioning and health related quality of life in older adults: a systematic review and meta-analysis. J Altern Complement Med. 2012;18(10):902-917. doi:10.1089/ acm.2011.0473.

44. Sobana R, Parthasarathy S, Duraisamy, Jaiganesh K, Vadivel S. The effect of yoga therapy on selected psychological variables among male patients with insomnia. J Clin Diagn Res. 2013;7(1):55-57. doi: 10.7860/JCDR/2012/5056.2669.

Copyright ( 2017 The Author (s); This is an open-access article distributed under the terms of the Creative Commons Attribution License (http://creativecommons.org/licenses/by/4.0), which permits unrestricted use, distribution, and reproduction in any medium, provided the original work is properly cited. 